\title{
Microspheres with Ultrahigh Holmium Content for Radioablation of Malignancies
}

\author{
W. Bult, ${ }^{1,7}$ P. R. Seevinck, ${ }^{2}$ G. C. Krijger, ${ }^{3}$ T. Visser, ${ }^{4}$ L. M. J. Kroon-Batenburg, ${ }^{5}$ C. J. G. Bakker, ${ }^{2}$ \\ W. E. Hennink, ${ }^{6}$ A. D. van het Schip, ${ }^{1}$ and J. F. W. Nijsen ${ }^{1}$
}

Received November 6, 2008; accepted February 5, 2009; published online February 25, 2009

\begin{abstract}
Purpose. The aim of this study was to develop microspheres with an ultra high holmium content which can be neutron activated for radioablation of malignancies. These microspheres are proposed to be delivered selectively through either intratumoral injections into solid tumors or administered via an intravascularly placed catheter.

Methods. Microspheres were prepared by solvent evaporation, using holmium acetylacetonate (HoAcAc) crystals as the sole ingredient. Microspheres were characterized using light and scanning electron microscopy, coulter counter, titrimetry, infrared and Raman spectroscopy, differential scanning calorimetry, X-ray powder diffraction, magnetic resonance imaging (MRI), and X-ray computed tomography $(\mathrm{CT})$.

Results. Microspheres, thus prepared displayed a smooth surface. The holmium content of the HoAcAc microspheres $(44 \%(w / w))$ was higher than the holmium content of the starting material, HoAcAc crystals $(33 \%(w / w))$. This was attributed to the loss of acetylacetonate from the HoAcAc complex, during rearrangement of acetylacetonate around the holmium ion. The increase of the holmium content allows for the detection of (sub)microgram amounts of microspheres using MRI and CT.

Conclusions. HoAcAc microspheres with an ultra-high holmium content were prepared. These microspheres are suitable for radioablation of tumors by intratumoral injections or treatment of liver tumors through transcatheter administration.
\end{abstract}

KEY WORDS: holmiumacetylacetonate; intratumoral; microspheres; multimodality; radioablation.

\section{INTRODUCTION}

Worldwide, primary liver cancer (hepatocellular carcinoma or cholangiocarcinoma) is the sixth most common cancer, and each year over 600,000 new cases are presented. A limited number of patients is eligible for surgical resection (partial hepatectomy or liver transplantation), limiting the 5 year survival rate to only $3-5 \%(1,2)$. The use of systemic

$\overline{{ }^{1} \text { Department of Radiology and Nuclear Medicine, University Medical }}$ Center Utrecht, P.O. Box 85500, 3508 GA Utrecht, The Netherlands.

${ }^{2}$ Image Sciences Institute, University Medical Center Utrecht, P.O. Box 85500, 3508 GA Utrecht, The Netherlands.

${ }^{3}$ Department of Radiation, Radionuclides and Reactors, Delft University of Technology, Mekelweg 15, 2629 JB Delft, The Netherlands.

${ }^{4}$ Inorganic Chemistry and Catalysis Group, Department of Chemistry, Faculty of Science, Utrecht University, P.O. Box 80083, 3508 TB Utrecht, The Netherlands.

${ }^{5}$ Department of Crystal and Structural Chemistry, Utrecht University, P.O. Box 80054, 3508 TB Utrecht, The Netherlands.

${ }^{6}$ Department of Pharmaceutics, Utrecht Institute for Pharmaceutical Sciences, Utrecht University, P.O. Box 80082, 3508 TB Utrecht, The Netherlands.

${ }^{7}$ To whom correspondence should be addressed. (e-mail: w.bult@um cutrecht.nl) chemotherapy has been proven ineffective, however recently a survival benefit of several months has been reported for the oral multikinase inhibitor sorafenib (3).

The liver is also a common site of metastasis, and more than $50 \%$ of patients with primary malignancies will develop hepatic metastases. A very common type of cancer to metastasize solely to the liver is colorectal carcinoma. Each year over 1,000,000 new cases of colorectal cancer are presented and the mortality is approximately 500,000 patients per year. Five year survival of patients in the developed world with colorectal cancer is approximately $55 \%$, with main prognostic factors being the extent of lymphatic spread, the number and resectability of liver metastases $(1,2)$. Surgical resection, considered to be the only curative treatment option, is only eligible in $10 \%$ to $20 \%$ of patients (4). Currently applied chemotherapy protocols (e.g. FOLFOX or FOLFORI, 5-fluorourcil/leucovorin combined with oxaliplatin or irrinotecan, respectively, in combination with the angiogenesis inhibitor bevacizumab) are associated with a survival of patients with unresectable liver metastases of nearly 2 years $(5,6)$.

Intra-arterial radioembolisation with radioactive microspheres (glass- or resin-based) containing the high-energy beta emitter yttrium-90 $\left({ }^{90} \mathrm{Y}\right)$ is currently increasingly applied in patients with unresectable hepatic malignancies. This ${ }^{90} \mathrm{Y}$ radioembolisation treatment has shown very high response 
rates (up to $91 \%$ ), in both salvage setting and as a first-line treatment option $(7,8)$. The lack of (quantitative) imaging options for ${ }^{90} \mathrm{Y}$ (9) has lead to the development of holmium166 loaded poly(L-lactic acid) $\left({ }^{166}\right.$ HoPLLA) microspheres, since

${ }^{166} \mathrm{Ho}$ emits gamma rays and beta particles that can be used for nuclear imaging and therapy, respectively (10-12). Moreover, holmium is highly paramagnetic and has a high linear attenuation coefficient allowing for visualization by magnetic resonance imaging (MRI) and X-ray computed tomography (CT), respectively $(13,14)$.

Unfortunately, restrictions to inclusion to this intraarterial transcatheter radioembolisation approach exist. For example, in patients who have been heavily pretreated with bevacizumab or cetuximab, the tumor feeding vessels may be too feeble for the microspheres to be deposited peri- and endotumorally (15). Efficacy may also be diminished in patients with hypovascular liver tumors. Furthermore, patients with portal vein thrombosis are excluded from treatment with the resin microspheres (16). Extensive liver cirrhosis and poor general health may also hamper the use of this treatment option.

Especially in patients with a limited number of unresectable intrahepatic tumors, not eligible for transcatheter radioembolisation, intratumoral injections of radioactive microspheres might be an option. This approach has been explored in patients with liver tumors using ${ }^{90} \mathrm{Y}$ glass microspheres, showing a response rate in $90 \%$ of patients (17). Direct intratumoral injection requires small deposits of radioactive microspheres in compact tumor tissue, limiting the overall injection volume to $0.1-0.3 \mathrm{ml}(17,18)$. Consequently, the amount of activity per injection should be very high.

The purpose of this study was to prepare holmium loaded microspheres, using holmium acetylacetonate (HoAcAc) crystals as the sole staring material, without the use of PLLA as matrix forming polymer, to increase the content of holmium per sphere in order to obtain microspheres suitable for intratumoral application.

\section{MATERIALS AND METHODS}

\section{Materials}

All chemicals are commercially available and were used as obtained. Acetylacetone (2,4-pentanedione, AcAc; >99\%), ammoniumhydroxide $\left(\mathrm{NH}_{4} \mathrm{OH} ; 29.3 \%\right.$ in water), chloroform (HPLC-grade), phosphorus pentoxide (Sicapent $\left.{ }^{\circledR}\right)$, polyvinyl alcohol (PVA, average MW 30,000-70,000) and Pluronic F68® $\left(\mathrm{PEO}_{100} \mathrm{PPO}_{65} \mathrm{PEO}_{100} ; \mathrm{MW} 9,840-14,600\right)$ were purchased from Sigma Aldrich (Steinheim, Germany). Holmium CertiPUR ICP standard and hexamethylentetramine were purchased from Merck (Darmstadt, Germany). Seakem LE Agarose was purchased from Lonza (Basel, Switzerland). Holmium (III) chloride hexahydrate $\left(\mathrm{HoCl}_{3} \cdot 6 \mathrm{H}_{2} 0 ; 99.95 \%\right)$ was purchased from Metall Rare Earth Ltd (Shenzhen, China).

\section{Preparation of HoAcAc Microspheres}

The holmium acetylacetonate microspheres (HoAcAc MS) were prepared using a solvent evaporation process. Holmium acetylacetonate crystals were prepared as previously described by Nijsen et al. (10). HoAcAc crystals were dissolved in chloroform (186 g, 5.5\% $(w / w))$ and emulsified in an aqueous PVA solution $(11,2 \%(w / w))$. The chloroform/ water emulsion was continuously stirred at a preset stirring speed (300-500 rpm). To evaporate chloroform, the mixture was thermostated at $25^{\circ} \mathrm{C}$ and a constant flow of nitrogen (12 $1 \mathrm{~min}^{-1}$ ) was applied. After $40 \mathrm{~h}$ of evaporation the formed microspheres were collected by centrifugation and washed five times with water. The washed microspheres were sieved using an electronic sieve vibrator, (EMS 755) and ultrasonic processor (UDS 751; Topaz GmbH, Dresden, Germany) as described by Zielhuis et al. (19). The microspheres were dried at room temperature for $24 \mathrm{~h}$, followed by drying at $50^{\circ} \mathrm{C}$ for $48 \mathrm{~h}$.

\section{Size Distribution, Holmium Content and Surface Characteristics}

The size distribution of the sieved fractions and dried fractions was determined using a Coulter counter (Multisizer 3, Beckman Coulter, Mijdrecht, The Netherlands), equipped with an orifice of $100 \mu \mathrm{m}$.

The holmium content of the microspheres was determined by complexometric titration with EDTA as described by Zielhuis et al. (19), and Inductively Coupled Plasma Optical Emission spectroscopy (ICP OES). Samples were measured at three different wavelengths (399.989; 345.600 and $347.426 \mathrm{~nm}$ ) in an Optima $4300 \mathrm{CV}$ (PerkinElmer; Norwalk, USA).

Elemental analysis of carbon, hydrogen, oxygen and holmium of the HoAcAc crystals and HoAcAc microspheres was performed by H. Kolbe, Mülheim an der Ruhr, Germany.

SEM micrographs were acquired using a FEI PHENOM (FEI Company, Eindhoven, The Netherlands) electron microscope. Samples were mounted on an aluminum stub, and coated with a Pt layer of $6 \mathrm{~nm}$.

\section{Raman Spectroscopy}

Raman spectroscopy was performed on a Kaiser RXN spectrometer equipped with a $70 \mathrm{~mW} 785 \mathrm{~nm}$ diode laser for excitation, a holographic grating for dispersion and a Peltier cooled Andor CCD camera for detection.

\section{Differential Scanning Calorimetry}

Modulated Differential Scanning Calorimetry (mDSC) measurements were performed using a DSC Q1000 (TA Instruments Inc., New Castle, DE, USA). The modulation amplitude was set at $1^{\circ} \mathrm{C} \mathrm{min}{ }^{-1}$. Samples were heated from $20^{\circ} \mathrm{C}$ to $220^{\circ} \mathrm{C}$ with a heating rate of $2^{\circ} \mathrm{C} \mathrm{min}^{-1}$.

\section{X-ray Diffraction}

X-ray diffraction patterns were obtained on a Nonius $\kappa$ $\mathrm{CCD}$ diffractometer, with a sealed tube using MoK $\alpha$ radiation with a graphite monochromator. Powder patterns were recorded to a maximum scattering angle $(2 \theta) 22^{\circ}$. The samples were contained in boroglass capillaries. 


\section{Neutron Activation}

Neutron activation was performed in the pneumatic rabbit system operational at a research reactor facility (Department of Radiation, Radionuclides and Reactors, Delft University of Technology, Delft, The Netherlands). Samples were irradiated for 3 or $6 \mathrm{~h}$ with a thermal neutron flux of $5 \times 10^{12} \mathrm{n} \mathrm{cm}^{-2} \mathrm{~s}^{-1}$. The radioactive ${ }^{166} \mathrm{Ho}$ was left to decay for 1 month, to ensure safe handling of HoAcAc microspheres.

\section{Multimodality Imaging Characteristics}

To determine the sensitivity of MRI and CT for the HoAcAc microspheres, phantom experiments were carried out on MRI and CT. The phantom used to determine MRI and CT sensitivity consisted of agarose $(2 \%(w / w))$ gel samples in which HoAcAc microspheres were homogeneously dispersed (loadings ranged from 0 to $4 \mathrm{mg}$ microspheres per $\mathrm{ml}$ agarose) in plastic tubes (diameter $10 \mathrm{~mm}$, length $100 \mathrm{~mm}$ ). MR imaging was performed using a $1.5 \mathrm{~T}$ clinical MR scanner (Intera, Philips Medical Systems, Best, The Netherlands). To determine the $r_{2} *$ relaxivity, which represents the MR sensitivity of the HoAcAc microspheres, transverse images were made using a multiple gradient echo MR sequence with 15 echos, an echo spacing of $1.39 \mathrm{~ms}$ a repetition time of $500 \mathrm{~ms}$, a field of view (FOV) of $128 \mathrm{~mm}$, a voxel size of $1 \times 1 \times 10 \mathrm{~mm}$, two signal averages and a $60^{\circ}$ flip angle. Monoexponential signal decay was assumed. Data processing was performed as described by Seevinck et al. (14). A second agarose gel phantom was constructed for qualitative imaging. This gel consisted of $2 \%(w / w)$ agarose, with pockets for the administration of a suspension of HoAcAc microspheres to obtain depots of $1 \mu \mathrm{g}$ to $1 \mathrm{mg}$. The imaging parameters were similar to the imaging parameters mentioned before, except for the slice thickness, which was $1 \mathrm{~mm}$.

To mimic potential in vivo imaging applications, depots of HoAcAc microspheres were injected in an ex vivo rabbit liver. The excised liver was flushed with water, and placed in an aqueous solution containing $\mathrm{MnCl}_{2}(19.2 \mathrm{mg} / \mathrm{L})$, to reduce the relaxation time of the water. Fifty microlitre of a suspension of HoAcAc microspheres was administered to the liver, to obtain depots of 1,5 and $10 \mu \mathrm{g}$ microspheres. The imaging parameters were similar to the imaging parameters mentioned before. For the MR images displayed in Fig. 5A, $\mathrm{D}, \mathrm{E}$ and $\mathrm{F}$ an echo time of $20 \mathrm{~ms}$ was used.

Quantitative X-ray CT imaging was performed on a clinical 64 slice CT scanner (Brilliance, Philips Medical Systems, Best, The Netherlands). The sample tubes were positioned co-axially to the main axis of the scanner. Using the methods described by Seevinck et al, a linear regression curve was constructed to determine the sensitivity of X-ray CT for HoAcAc microspheres (14).

\section{RESULTS AND DISCUSSION}

\section{Size Distribution, Holmium Content and Surface Characteristics}

We found that HoAcAc microspheres could be obtained of HoAcAc crystals without using PLLA as a matrix forming polymer using a solvent evaporation technique.

The size distribution, surface morphology of the microspheres were characterized using Coulter Counter, light microscopy and scanning electron microscopy (SEM), both before and after neutron activation. The particle size distribution was rather broad, from 5 to $40 \mu \mathrm{m}$, with an average microsphere size of $15 \mu \mathrm{m}$ when the microspheres were prepared at $500 \mathrm{rpm}$. The average particle size could be tailored by altering the stirring rate during emulsifying and increased from $15 \mu \mathrm{m}$ at $500 \mathrm{rpm}$ to $20 \mu \mathrm{m}$ at $400 \mathrm{rpm}$ and $26 \mu \mathrm{m}$ at $300 \mathrm{rpm}$. The size distribution should be narrow to control the biodistribution of the microspheres and to reduce possible shunting of small microspheres to non-target organs after administration. Therefore, a sieving step is necessary to obtain a more confined size distribution. More than $93 \%$ of the microspheres had a size between 15-20 $\mu \mathrm{m}$ after sieving. No changes in microsphere size distribution were observed after neutron activation, which is an indication that small fragments due to radiation damage were absent. Light and scanning electron microscopy showed that spherical particles with a smooth surface were formed (Fig. 1A and B). Neither surface damage nor agglomeration was observed after neutron activation of the microspheres. After neutron activation for 3 or even $6 \mathrm{~h}$, the surface of the microspheres only showed small crevices. However, no large fragments were present (Fig. 1C). This, in combination with the size
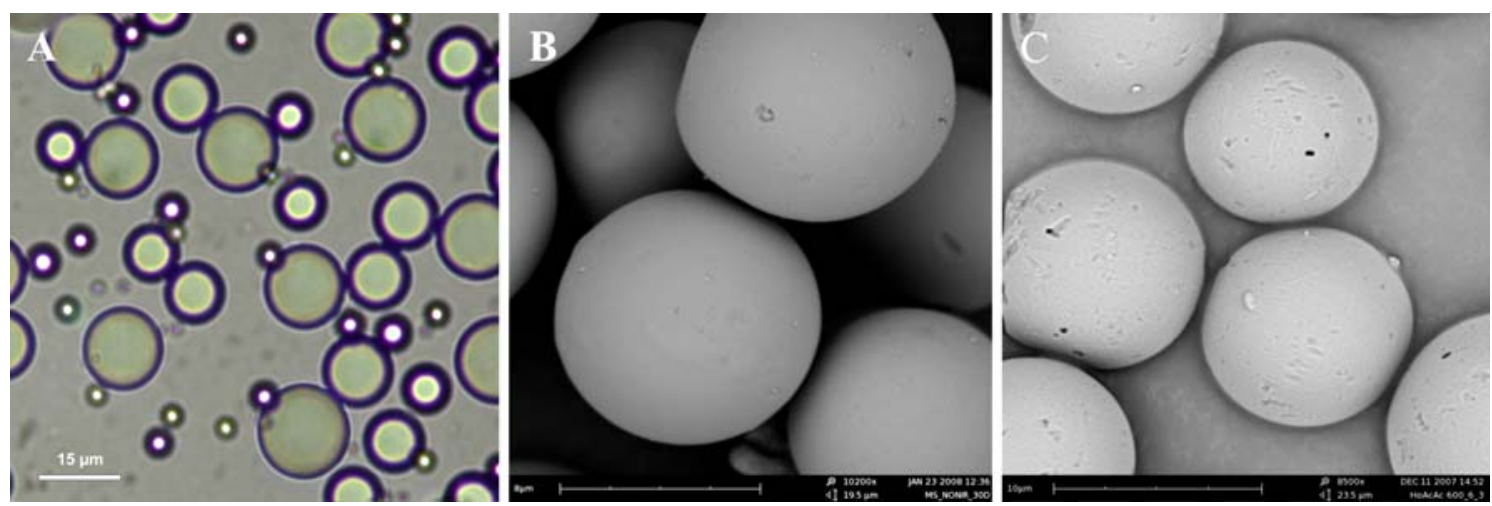

Fig. 1. A Light micrograph of HoAcAc microspheres before sieving (mean size $15 \mu \mathrm{m}$ ), B scanning electron micrograph of non activated HoAcAc microspheres and $\mathbf{C}$ scanning electron micrograph of HoAcAc microspheres after $6 \mathrm{~h}$ of neutron activation. 
Table I. Calculated and Measured Elemental Composition of HoAcAc Crystals and Proposed and Measured Composition of HoAcAc Microspheres (in $\% w / w)$

\begin{tabular}{|c|c|c|c|c|}
\hline \multirow[b]{2}{*}{ Element } & \multicolumn{2}{|c|}{ HoAcAc Crystals $\mathrm{Ho}(\mathrm{AcAc})_{3} \bullet 3 \mathrm{H}_{2} \mathrm{O}$} & \multicolumn{2}{|c|}{ HoAcAc Microspheres $\mathrm{Ho}_{2}(\mathrm{AcAc})_{3} \bullet 4 \mathrm{H}_{2} \mathrm{O}$} \\
\hline & Calculated & Measured $^{a}$ & Proposed $^{b}$ & Measured \\
\hline $\mathrm{C}$ & 34.9 & 36.5 & 25.8 & 27.0 \\
\hline $\mathrm{H}$ & 5.3 & 5.0 & 4.2 & 3.8 \\
\hline $\mathrm{O}$ & 27.9 & 27.1 & 22.9 & 24.2 \\
\hline Ho & 31.9 & 31.2 & 47.2 & 45.0 \\
\hline
\end{tabular}

${ }^{a}$ Data taken from Nijsen et al. (10)

${ }^{b}$ Structure of HoAcAc microspheres is proposed based on measured elemental composition and Raman spectroscopy

distribution results, indicates that the microspheres are resistant to neutron irradiation.

The holmium content of the HoAcAc microspheres, as determined by complexometric titration, ICP OES and elemental analysis was found to be $45.0 \pm 0.5 \%(w / w)$, which is significantly higher than the holmium content of the HoAcAc crystals, being $31.2 \%(w / w)$ (Table I). Also, the density of the HoAcAc microspheres $(1.90 \mathrm{~g} / \mathrm{ml})$ was higher than the density reported for HoAcAc crystals $(1.77 \mathrm{~g} / \mathrm{ml}$ (20)). In the HoAcAc crystals, holmium is surrounded by three acetylacetonate ligands, and three water molecules $(10,20)$. The increase in holmium content of the HoAcAc microspheres was explained by a loss of one or more acetylacetonate ligands caused by a structural rearrangement of the acetylacetonate ligands around holmium. This hypothesis was further investigated by determining the elemental composition of the microspheres and Raman spectroscopy.

The substantially higher holmium content per sphere $(17.0 \%(w / w)$ for the HoPLLA microspheres $v s .45 .0 \%(w / w)$ for the HoAcAc microspheres) results in a significant increase in radioactivity per sphere from $29 \mathrm{MBq} / \mathrm{mg}$ for HoPLLA microspheres (12) to $76 \mathrm{MBq} / \mathrm{mg}$ for HoAcAc microspheres $(6 \mathrm{~h}$ neutron activation; thermal neutron flux of $\left.5 \times 10^{12} \mathrm{n} \mathrm{cm}^{-2} \mathrm{~s}^{-1}\right)$. Clinical results after intratumoral injection of ${ }^{90} \mathrm{Y}$ glass microspheres showed that $74 \mathrm{MBq}{ }^{90} \mathrm{Y}$ is sufficient to evoke a tumoricidal effect on a tumor of $3 \mathrm{~cm}$ (17). The injected volume of the microsphere suspension to such a tumor is limited to typically $0.1-0.3 \mathrm{ml}$ (18). Based on the radiation characteristics of ${ }^{166} \mathrm{Ho}$ and ${ }^{90} \mathrm{Y}$ it can be calculated that a tumoricidal dose of ${ }^{166} \mathrm{Ho}$ activity (230 MBq/3 mg) can be delivered in this volume using HoAcAc microspheres for intratumoral administration (12).

\section{Raman Spectroscopy}

Infrared and Raman spectroscopy was performed to ascertain the presence of acetylacetonate in the microspheres. The Raman spectra of the HoAcAc crystals and the HoAcAc microspheres were virtually identical (Fig. 2), which implies that holmium is surrounded by acetylacetonate in both the HoAcAc crystals and the HoAcAc microspheres. These findings are in agreement with the infrared measurements on HoAcAc microspheres that showed the same peak characteristics as for HoAcAc crystals previously reported (21). The increase of the holmium content, found by elemental analysis, complexometric titration and ICP OES can therefore only be explained by a rearrangement of acetylacetonate groups around the holmium(III) ion. Taking into account that holmium is surrounded by acetylacetonate ligands, and the results from the elemental analysis (Table I), we propose that per holmium ion one and a half acetylacetonate and two water molecules are present in the HoAcAc microspheres. The change of the acetylacetonate coordination should, in principle, be reflected in an intensity decrease of the Ho-O bending band, but since the position of this vibration is expected around $100 \mathrm{~cm}^{-1}$ (22), it is most likely filtered out with the Rayleigh scattering. The hypothesized structure of the HoAcAc microspheres is depicted in Fig. 3. As can be seen in this figure, acetylacetonate forms a bridge between two holmium(III) ions by coordinating each carbonyl to another holmium(III) ion forming a network. This bridging effect of chelates between metal ions has been proposed for metal catalysts after gradual removal of the solvent (23). The water observed in elemental analysis (Table I) is likely coordinated to the holmium(III) ion, as previously reported for HoAcAc crystals (20). An important observation that supports the network formation between holmium and acetylacetonate is that HoAcAc microspheres, in contrast to HoAcAc crystals, did not dissolve in chloroform.

\section{Differential Scanning Calorimetry}

The thermal behaviour of HoAcAc crystals and HoAcAc microspheres was investigated using modulated differential scanning calorimetry (mDSC). Representative mDSC scans of the holmium acetylacetonate crystals and microspheres are shown in Fig. 4, and relevant data are summa-

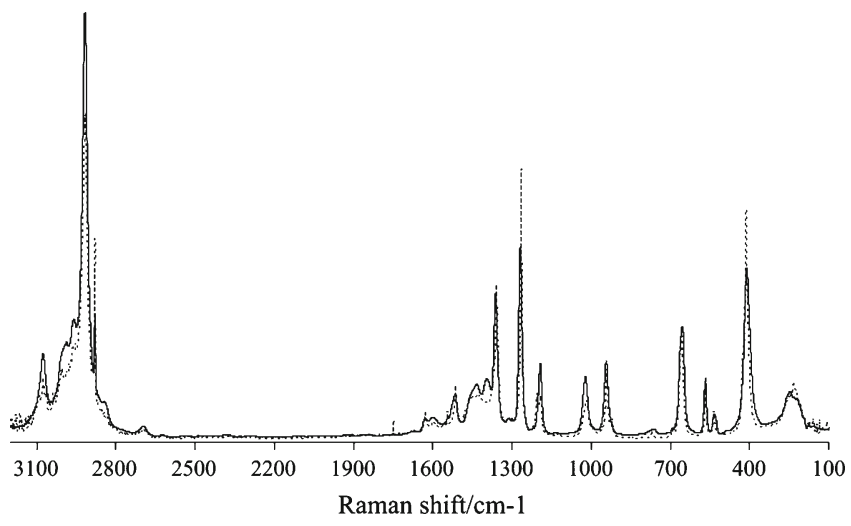

Fig. 2. Raman spectra of HoAcAc microspheres (solid line) and HoAcAc crystals (dotted line). 


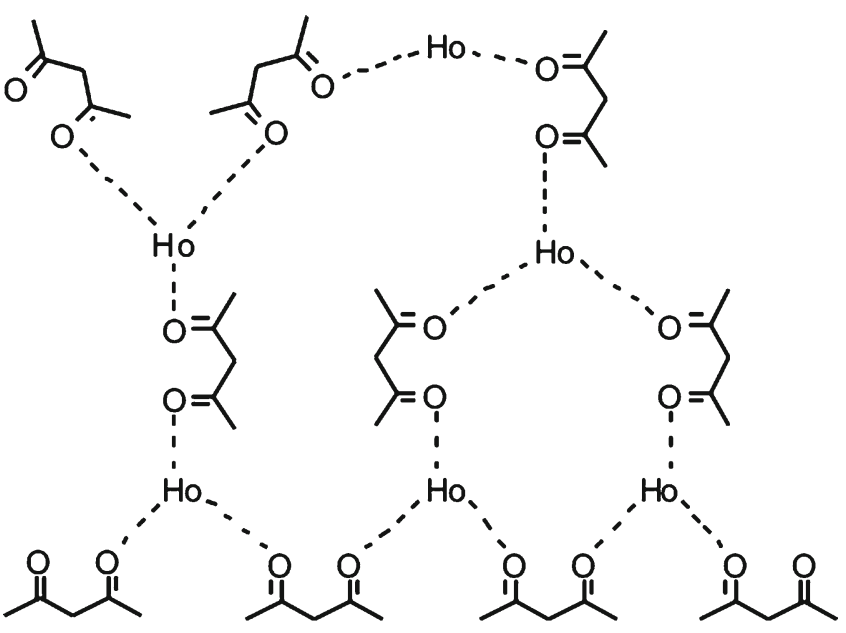

Fig. 3. The proposed interaction of acetylacetonate with holmium (III) ion in HoAcAc microspheres (coordinated water molecules not shown). Each acetylacetonate carbonyl oxygen coordinates with a separate holmium(III) ion, linking two holmium(III) ions.

rized in Table II. The thermogram for HoAcAc crystals shows an endotherm with a peak maximum at $123 \pm 7^{\circ} \mathrm{C}$. This endotherm was ascribed to the loss of water from the complex, leading to the collapse of the crystalline structure of the crystals (21). HoAcAc microspheres show a distinctly different thermogram. We observed an endotherm at approximately $194 \pm 2^{\circ} \mathrm{C}$, which is likely due to the loss of water from the structure. Interestingly, after heating to $200^{\circ} \mathrm{C}$ the microspheres were still intact, and the surface of the microspheres showed minor signs of damage. In contrast, heating of the HoAcAc crystals to $200^{\circ} \mathrm{C}$ lead to completely molten material, which is in agreement with literature (24). The resistance of microspheres to heating to $200^{\circ} \mathrm{C}$ is also an indication that a network is formed. Moreover, this resistance to heat allows for dry heat sterilization (25), making the microspheres suitable for in vivo use. DSC analysis of neutron activated HoAcAc microspheres showed an endotherm at $199 \pm 3^{\circ} \mathrm{C}$, which is likely associated with the loss of water, which was also observed for the non activated microspheres.

\section{$X$-ray Diffraction}

The internal structure of the HoAcAc crystals and HoAcAc microspheres was studied using X-ray diffraction. The HoAcAc crystals have a crystalline structure (21), with a powder pattern reflecting the periodicity of the crystal structure. The pattern for the HoAcAc microspheres shows no sharp peaks at a higher diffraction angle. This indicates the absence of repetitive intermolecular interactions, which is typical for amorphous structures. The broad peak in the HoAcAc microsphere diffraction pattern at a diffraction angle of approximately $11^{\circ}$ can be attributed to the distance of two holmium atoms separated by one acetylacetonate molecule, which is approximately $4.5 \AA$. Since holmium has a large scattering power due to the relatively high number of electrons, the high intensity peak at a diffraction angle of approximately $3.2^{\circ}$ is ascribed to the Ho-Ho contact in its amorphous form, corresponding to a bond length of approximately $15.5 \AA$ (21).

\section{Multimodality Imaging Characteristics}

The paramagnetic nature and the high linear attenuation coefficient of holmium allows for multimodality imaging of non-radioactive HoPLLA microspheres.

To investigate if the multimodality imaging characteristics enhanced with an increase of the holmium concentration, the HoAcAc microspheres were homogeneously dispersed in an agarose gel matrix. The paramagnetic nature of holmium locally induces a magnetic field inhomogeneity, leading to an enhanced MRI signal decay in gradient echo images (13). The resulting $r_{2}{ }^{*}$ relaxivity of the HoAcAc microspheres with a holmium load of $45 \%(w / w)$, is $264 \pm$ $5.7 \mathrm{~s}^{-1} \mathrm{mg}^{-1} \mathrm{ml}$, which is substantially higher than the $r_{2}{ }^{*}$ value for the HoPLLA microspheres with a holmium load of $17 \%(w / w)\left(92 \pm 3.2 \mathrm{~s}^{-1} \mathrm{mg}^{-1} \mathrm{ml}\right)(26)$. This increase of the $r_{2}{ }^{*}$ relaxivity represents an enhanced sensitivity of MRI to the HoAcAc microspheres, as compared with HoPLLA microspheres, which can be explained by the increased holmium weight content per sphere.

Quantitative CT of the agarose phantom resulted in a mass attenuation coefficient at $120 \mathrm{kV}$ of $15.6 \mathrm{HU} \mathrm{mg}^{-1} \mathrm{ml}$ for HoAcAc MS, which is substantially increased, when compared to the mass attenuation coefficient of HoPLLA microspheres of 6.7 $\mathrm{HU} \mathrm{mg}^{-1} \mathrm{ml}$ at $120 \mathrm{kV}$ (14). This increase in CT sensitivity, like the increase of sensitivity of MRI to the HoAcAc microspheres, can be attributed to the increase in holmium content.

To demonstrate the feasibility of visualization of microgram amounts of HoAcAc microspheres, using MRI and CT, decreasing amounts of HoAcAc microspheres $(1 \mathrm{mg}$ to $1 \mu \mathrm{g}$ ) in clusters were imaged in vitro as well as ex-vivo. The enhanced $r_{2} *$ relaxivity of the HoAcAc microspheres allowed for the detection of $1 \mu \mathrm{g}$ of HoAcAc microspheres in the gel (Fig. 5A). The same setup was used for CT measurements, and the increased Ho content per sphere resulted in detection

Table II. Summary of DSC Data of HoAcAc Crystals and HoAcAc Microspheres Both Non Neutron Activated and Neutron Activated $n=6-8$

\begin{tabular}{lccc}
\hline & Onset $T_{\max }\left({ }^{\circ} \mathrm{C}\right)^{a}$ & $T_{\max }\left({ }^{\circ} \mathrm{C}\right)^{b}$ & $\Delta H(\mathrm{~J} / \mathrm{g})^{c}$ \\
\hline HoAcAc Crystals & $118 \pm 7$ & $123 \pm 7$ & $115 \pm 9$ \\
HoAcAc Microspheres & $188 \pm 2$ & $194 \pm 2$ & $25 \pm 5$ \\
Neutron activated HoAcAc Microspheres & $190 \pm 3$ & $199 \pm 3$ & $12 \pm 11$ \\
\hline
\end{tabular}

${ }^{a}$ Onset $T_{\max }$ : onset of the endotherm

${ }^{b} T_{\text {max }}$ : peak maximum of the endotherm

${ }^{c} \Delta H$ : enthalpy of the endotherm 


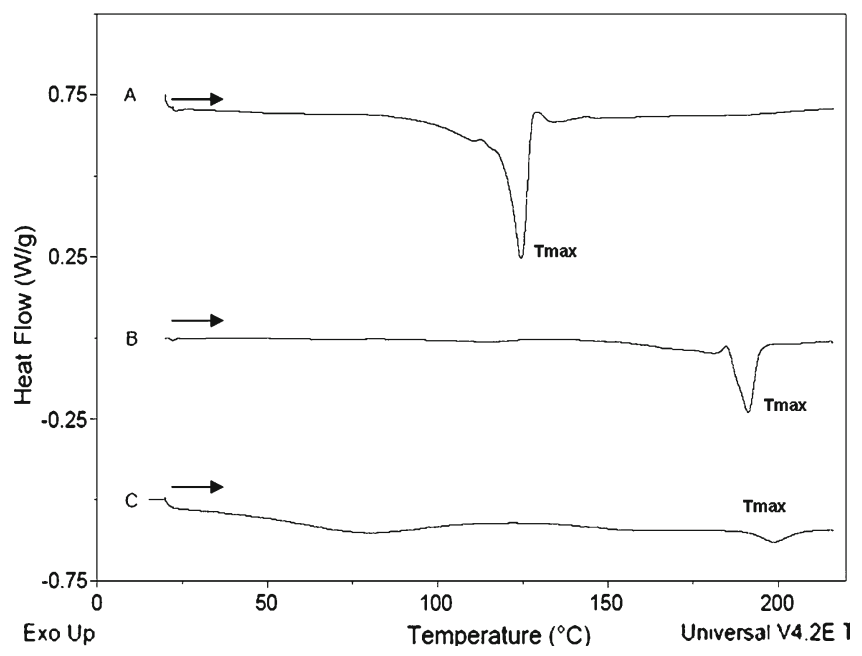

Fig. 4. mDSC thermograms of $\mathbf{A}$ holmium acetylacetonate crystals, $\mathbf{B}$ holmium acetylacetonate microspheres and $\mathbf{C}$ neutron activated $(6 \mathrm{~h})$ holmium acetylacetonate microspheres. $\mathrm{T}_{\max }$ is the maximum peak of the endotherm.

of $50 \mu \mathrm{g}$ HoAcAc microspheres (Fig. 5B and C). We used an excised rabbit liver (Fig. 5D), with depots of 1,5 and $10 \mu \mathrm{g}$ HoAcAc microspheres to investigate the ex-vivo imaging characteristics on MRI. The inhomogeneity of the ex-vivo rabbit liver resulted in the detection of depots from $5 \mu \mathrm{g}$ onwards (Fig. 5E and F).
Unlike the ${ }^{90} \mathrm{Y}$ microspheres, which lack the MR imaging possibilities, holmium microspheres can be visualized directly therefore allow for tracking of the microspheres long after the radioactive ${ }^{166} \mathrm{Ho}$ has decayed, and thus establish the in vivo fate of the microspheres after intratumoral injection in a non invasive manner using MRI. The detection of microgram amounts of HoAcAc microspheres will also enable MRI guided administration of the particles, allowing for accurate positioning of the depots in tumor tissue, whilst refraining healthy tissue from radiation damage.

\section{Clinical Perspective}

The two currently available ${ }^{90} \mathrm{Y}$ microspheres are glassbased (Therasphere ${ }^{\circledR}$, MDS Nordion, Kanata, Canada) or resin-based (SIR-Spheres ${ }^{\circledR}$, SIRTeX Medical Ltd., Sydney, Australia). The glass microspheres have a very high specific activity (2.5 kBq/microsphere), and are registered as a Humanitarian Use Device by the FDA for use in radiation treatment or neoadjuvant to surgery or transplantation in patients with unresectable hepatocellular carcinoma (27). The resin based microspheres have a relatively low specific activity $40 \mathrm{~Bq} /$ sphere, and were granted premarket approval by the FDA in 2002 (27).

With regard to the transcatheter approach, the HoAcAc microspheres might be considered for treatment of patients with hypovascular liver malignancies. This is due to the specific activity of the ${ }^{166} \mathrm{HoAcAc}$ microspheres which is in
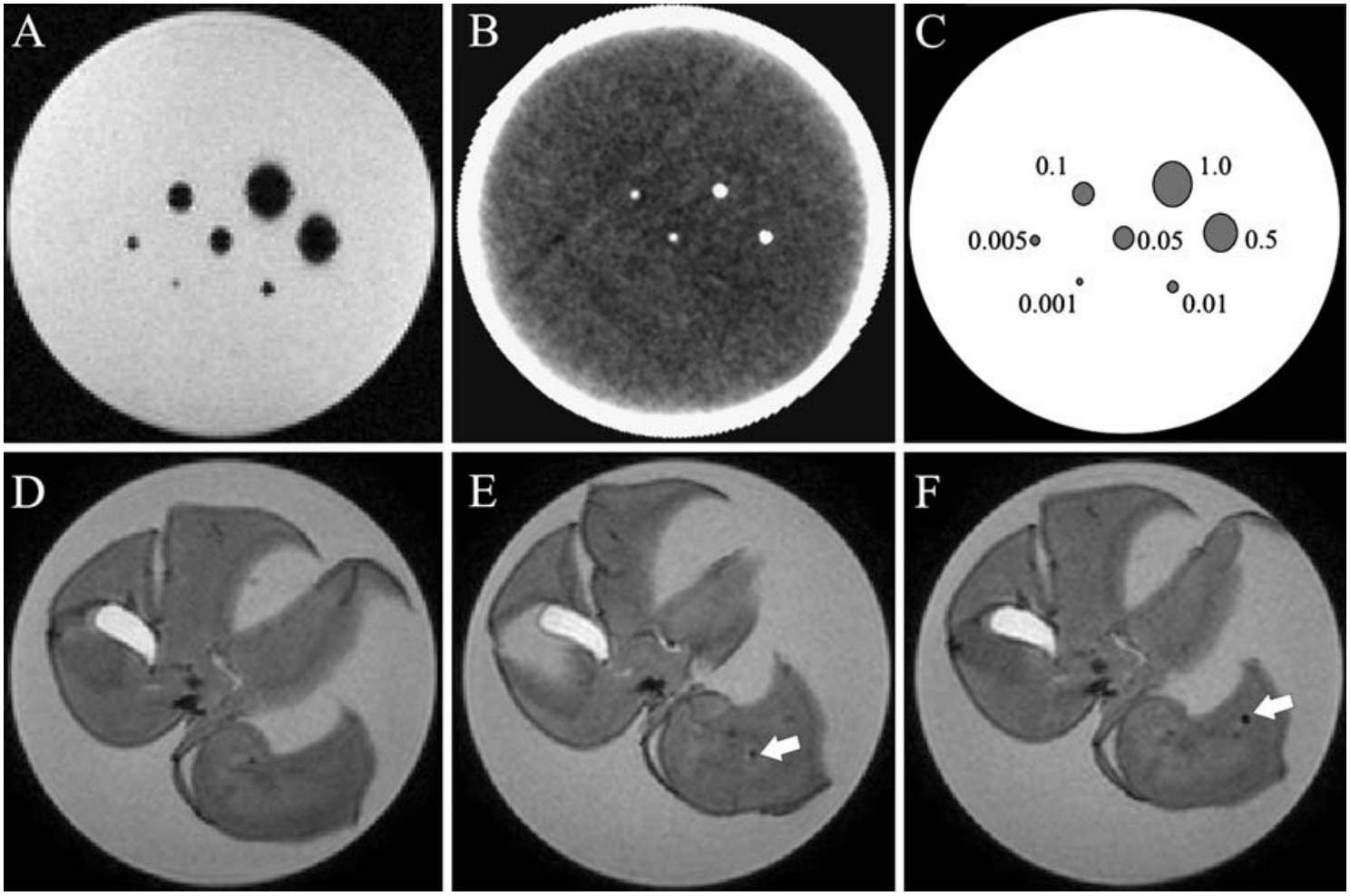

Fig. 5. A Gradient echo MRI image of agarose phantom with different depots of HoAcAc microspheres, B CT image of agarose phantom with different depots of HoAcAc microspheres, $\mathbf{C}$ schematic representation of agarose phantom with different depots of HoAcAc microspheres, including the amount of microspheres in milligram, D MRI image of rabbit liver before administration of HoAcAc microspheres, E MRI image of rabbit liver after administration of $5 \mu \mathrm{g}$ HoAcAc microspheres, indicated by arrow, $\mathbf{F}$ MRI image of rabbit liver after administration of $10 \mu \mathrm{g}$ HoAcAc microspheres, indicated by arrow. 
the range of the specific activity of the glass ${ }^{90} \mathrm{Y}$ microspheres. The obvious advantage of the ${ }^{166} \mathrm{HoAcAc}$ microspheres lies in multimodality imaging.

Moreover, the high specific activity of the ${ }^{166} \mathrm{HoAcAc}$ microspheres will allow for the delivery of a tumoricidal dose through intratumoral injections. This approach is thought to be applicable not only in patients with liver malignancies, but also in patients with solid tumors in other organs, e.g. brain, kidney and lungs.

However, before a phase I clinical study is allowed to be conducted, several issues need to be addressed. First the pharmaceutical quality needs to be investigated, e.g. stability testing. Secondly, the in vivo toxicity needs to be assessed. The toxicity is expected to be minor, since holmium is a relatively non toxic metal (28). Finally, the therapeutic efficacy of the ${ }^{166} \mathrm{HoAcAc}$ microspheres needs to be evaluated in tumor bearing animals.

\section{CONCLUSION}

In conclusion, the present study demonstrates that microspheres with an ultra high holmium content can be prepared from HoAcAc crystals as the sole starting material, without the use of a polymeric matrix, using a solvent evaporation process. The resulting microspheres have a smooth surface, and are resistant to neutron activation with a thermal neutron flux $\left(5 \times 10^{12} \mathrm{n} \mathrm{cm}^{-2} \mathrm{~s}^{-1}\right)$ and heating to $200^{\circ} \mathrm{C}$. The mean particle size can be tailored for different tumor treatment approaches by adapting the processing parameters. The size increased from 15 to $26 \mu \mathrm{m}$ when lowering the stirring rate from 500 to $300 \mathrm{rpm}$ during emulsifying. The HoAcAc microspheres have an unexpectedly high holmium content of $45 \%(w / w)$ which is substantially higher when compared to the HoPLLA microspheres $(17 \%(w / w))$. Moreover, the holmium content in HoAcAc microspheres is higher than in the HoAcAc crystals $(31 \%(w / w))$, which is attributed to a rearrangement of acetylacetonate molecules around the holmium(III) ion, resulting in the loss of one and a half coordinating acetylacetonate molecules per holmium(III) ion. The data of this study suggest that acetylacetonate acts as a bridge between holmium(III) ions, forming an amorphous network. The increased holmium content leads to a 2.6 fold higher radioactivity per sphere $(29 \mathrm{MBq} / \mathrm{mg}$ for HoPLLA microspheres vs. $76 \mathrm{MBq} / \mathrm{mg}$ for HoAcAc microspheres), which allows for tumoricidal radioactive doses in small volumes necessary for direct intratumoral injection. Additionally, the increased holmium content leads to a higher sensitivity for MRI and CT imaging, enabling detection of microgram amounts of microspheres in vivo.

\section{ACKNOWLEDGEMENTS}

This research was financially supported by the Dutch Technology Foundation STW under grant UGT 6069. We thank mr. R. de Roos and mr. A.J. de Graaf M.Sc. for their assistance in preparing the microspheres, Dr. W.A.M. van Maurik and Dr. W.H. Muller from EMSA, Faculty of Biology Utrecht University for assistance in scanning electron microscopy image acquisition and mr. M.J. van Steenbergen for his assistance with the DSC measurements.
Open Access This article is distributed under the terms of the Creative Commons Attribution Noncommercial License which permits any noncommercial use, distribution, and reproduction in any medium, provided the original author(s) and source are credited.

\section{REFERENCES}

1. F. Kamangar, G. M. Dores, and W. F. Anderson. Patterns of cancer incidence, mortality, and prevalence across five continents: defining priorities to reduce cancer disparities in different geographic regions of the world. J. Clin. Oncol. 24:2137-2150 (2006) doi:10.1200/JCO.2005.05.2308.

2. D. M. Parkin, F. Bray, J. Ferlay, and P. Pisani. Global cancer statistics, 2002. Ca Cancer J. Clin. 55:74-108 (2005) doi:10.3322/ canjclin.55.2.74.

3. J. M. Llovet, and J. Bruix. Molecular targeted therapies in hepatocellular carcinoma. Hepatology. 48:1312-1327 (2008) doi:10.1002/hep.22506.

4. E. Vibert, L. Canedo, and R. Adam. Strategies to treat primary unresectable colorectal liver metastases. Semin. Oncol. 32:33-39 (2005) doi:10.1053/j.seminoncol.2005.07.015.

5. H. S. Hochster, L. L. Hart, R. K. Ramanathan, B. H. Childs, J. D. Hainsworth, A. L. Cohn, L. Wong, L. Fehrenbacher, Y. Abubakr, M. W. Saif, L. Schwartzberg, and E. Hedrick. Safety and efficacy of oxaliplatin and fluoropyrimidine regimens with or without bevacizumab as first-line treatment of metastatic colorectal cancer: results of the TREE Study. J. Clin. Oncol. 26:3523-3529 (2008) doi:10.1200/JCO.2007.15.4138.

6. D. S. Zuckerman, and J. W. Clark. Systemic therapy for metastatic colorectal cancer: current questions. Cancer. 112:1879-1891 (2008) doi:10.1002/cncr.23409.

7. M. A. D. Vente, M. Wondergem, I. van der Tweel, M. A. A. J van den Bosch, B. A. Zonnenberg, M. G. E. H. Lam, A. D. van het Schip, and J. F. W. Nijsen. Yttrium-90 microsphere radioembolization for the treatment of liver malignancies: a structured meta-analysis. Eur. Radiol. 2008 doi:10.1007/s00330-008-1211-7.

8. G. van Hazel, A. Blackwell, J. Anderson, D. Price, P. Moroz, G. Bower, G. Cardaci, and B. Gray. Randomised phase 2 trial of SIR-Spheres plus fluorouracil/leucovorin chemotherapy versus fluorouracil/leucovorin chemotherapy alone in advanced colorectal cancer. J. Surg. Oncol. 88:78-85 (2004) doi:10.1002/ jso. 20141.

9. T. Gupta, S. Virmani, T. M. Neidt, B. Szolc-Kowalska, K. T. Sato, R. K. Ryu, R. J. Lewandowski, V. L. Gates, G. E. Woloschak, R. Salem, R. A. Omary, and A. C. Larson. MR Tracking of Ironlabeled Glass Radioembolization Microspheres during Transcatheter Delivery to Rabbit VX2 Liver Tumors: Feasibility Study. Radiology. 249(3):845-854 (2008).

10. J. F. W. Nijsen, B. A. Zonnenberg, J. R. Woittiez, D. W. Rook, I. A. Swildens-van Woudenberg, P. P. van Rijk, and A. D. van het Schip. Holmium-166 poly lactic acid microspheres applicable for intraarterial radionuclide therapy of hepatic malignancies: effects of preparation and neutron activation techniques. Eur. J. Nucl. Med. 26:699-704 (1999) doi:10.1007/s002590050440.

11. S. W. Zielhuis, J. F. W. Nijsen, J. H. Seppenwoolde, C. J. G. Bakker, G. C. Krijger, H. F. Dullens, B. A. Zonnenberg, P. P. van Rijk, W. E. Hennink, and A. D. van het Schip. Long-term toxicity of holmiumloaded poly(L-lactic acid) microspheres in rats. Biomaterials. 28:4591-4599 (2007) doi:10.1016/j.biomaterials.2007.07.012.

12. M. A. Vente, J. F. Nijsen, T. C. de Wit, J. H. Seppenwoolde, G. C. Krijger, P. R. Seevinck, A. Huisman, B. A. Zonnenberg, T. S. G. A. M. Van den Ingh, and A. D. van het Schip. Clinical effects of transcatheter hepatic arterial embolization with holmium-166 poly(L: -lactic acid) microspheres in healthy pigs. Eur. J. Nucl. Med. Mol. Imaging. 35:1259-1271 (2008) doi:10.1007/s00259-008-0747-8.

13. S. Fossheim, C. Johansson, A. K. Fahlvik, D. Grace, and J Klaveness. Lanthanide-based susceptibility contrast agents: assessment of the magnetic properties. Magn. Reson. Med. 35:201206 (1996) doi:10.1002/mrm.1910350211. 
14. P. R. Seevinck, J. H. Seppenwoolde, T. C. de Wit, J. F. W. Nijsen, F. J. Beekman, A. D. van het Schip, and C. J. G. Bakker. Factors affecting the sensitivity and detection limits of MRI, CT, and SPECT for multimodal diagnostic and therapeutic agents. Anticancer Agents Med Chem. 7:317-334 (2007) doi:10.2174/ 187152007780618153.

15. Kennedy, A. S. Dosimetry Update, Presented at the emerging trends in radioembolisation using microspheres, clinical workshop, Chicago, IL, 4-5 May 2007.

16. L. M. Kulik, B. I. Carr, M. F. Mulcahy, R. J. Lewandowski, B. Atassi, R. K. Ryu, K. T. Sato, A. Benson III, A. A. Nemcek Jr., V. L. Gates, M. Abecassis, R. A. Omary, and R. Salem. Safety and efficacy of $90 \mathrm{Y}$ radiotherapy for hepatocellular carcinoma with and without portal vein thrombosis. Hepatology. 47:71-81 (2008) doi:10.1002/hep.21980.

17. J. H. Tian, B. X. Xu, J. M. Zhang, B. W. Dong, P. Liang, and X. D. Wang. Ultrasound-guided internal radiotherapy using yttrium-90-glass microspheres for liver malignancies. J. Nucl. Med. 37:958-963 (1996).

18. W. Y. Lin, S. C. Tsai, J. F. Hsieh, and S. J. Wang. Effects of 90Ymicrospheres on liver tumors: comparison of intratumoral injection method and intra-arterial injection method. $J \mathrm{Nucl}$ Med. 41:1892-1897 (2000).

19. S. W. Zielhuis, J. F. W. Nijsen, R. Figueiredo, B. Feddes, A. M. Vredenberg, A. D. van het Schip, and W. E. Hennink. Surface characteristics of holmium-loaded poly(l-lactic acid) microspheres. Biomaterials. 26:925-932 (2005) doi:10.1016/j.biomaterials.2004.03.028.

20. H. Kooijman, F. Nijsen, A. L. Spek, and A. D. van het Schip. Diaquatris(pentane-2,4-dionato-O, $\mathrm{O}^{\prime}$ )holmium(III) monohydrate and diaquatris(pentane-2,4-dionato- $O, O^{\prime}$ )holmium(III) 4-hydroxypentan-2-one solvate dehydrate. Acta Crystallogr C. 56:156-158 (2000) doi:10.1107/S0108270199013566.
21. J. F. W. Nijsen, M. J. van Steenbergen, H. Kooijman, H. Talsma, L. M. Kroon-Batenburg, M. van de Weert, P. P. van Rijk, A. De Witte, A. D. van het Schip, and W. E. Hennink. Characterization of poly(L-lactic acid) microspheres loaded with holmium acetylacetonate. Biomaterials. 22:3073-3081 (2001) doi:10.1016/ S0142-9612(01)00055-2.

22. A. M. A. Bennett, G. A. Foulds, and D. A. Thornton. The Ir and H-1, C-13 Nmr-Spectra of the Nickel(II), Copper(II) and Zinc(II) Complexes of 2,4-Pentanedione, 4-Imino-2-Pentanone, 4-Thioxo-2-Pentanone and 2,4-Pentanedithione-A Comparative-Study. Polyhedron. 8:2305-2311 (1989) doi:10.1016/S02775387(00)81258-9.

23. A. J. Dillen, R. J. A. M. Terode, D. J. Lensveld, J. W. Geus, and K. P. de Jong. Synthesis of supported catalysts by impregnation and drying using aqueous chelated metal complexes. J. Catal. 216:257-264 (2003) doi:10.1016/s0021-9517(02)00130-6.

24. G. W. Pope, J. F. Steinbach, and W. F. Wagner. Characteristics of the solvates of the rare-earth acetylacetonates. J. Inorg. Nucl. Chem. 20:304-313 (1961) doi:10.1016/0022-1902(61)80281-9.

25. European Pharmacopoeia, $6^{\text {th }}$ Edition, Supplement 6.1, Directorate for the Quality of Medicines \& HealthCare of the Council of Europe, Strassbourgh, 2008.

26. J. F. W. Nijsen, J. H. Seppenwoolde, T. Havenith, C. Bos, C. J. G. Bakker, and A. D. van het Schip. Liver tumors: MR imaging of radioactive holmium microspheres-phantom and rabbit study. Radiology. 231:491-499 (2004) doi:10.1148/radiol.2312030594.

27. M. A. D. Vente, M. G. G. Hobbelink, A. D. van het Schip, B. A. Zonnenberg, and J. F. W. Nijsen. Radionuclide liver cancer therapies: from concept to current clinical status. Anticancer Agents Med Chem. 7:441-459 (2007) doi:10.2174/187152007781058569.

28. P. Arvela. Toxicity of rare-earths. Prog. Pharmacol. 2:73-113 (1979). 